\title{
A Hybrid Method for Powdered Materials Modeling
}

\author{
Yang Gao \\ 1. Research Institute of Frontier \\ Science, \\ 2. State Key Laboratory of Virtual \\ Reality Technology and Systems, \\ Beihang University, China.
}

\author{
Yinghao $\mathrm{Xu}^{*}$ \\ State Key Laboratory of Virtual \\ Reality Technology and Systems, \\ Beihang University, China.
}

\author{
Shuai Li \\ 1.State Key Laboratory of Virtual \\ Reality Technology and Systems, \\ 2.Beihang University Qingdao \\ Research Institute, Beihang \\ University, China.
}

\begin{abstract}
Aimin Hao
State Key Laboratory of Virtual

Reality Technology and Systems,

Beihang University, China.
\end{abstract}

\author{
Hong Qin \\ Stony Brook University, Stony Brook, \\ USA.
}
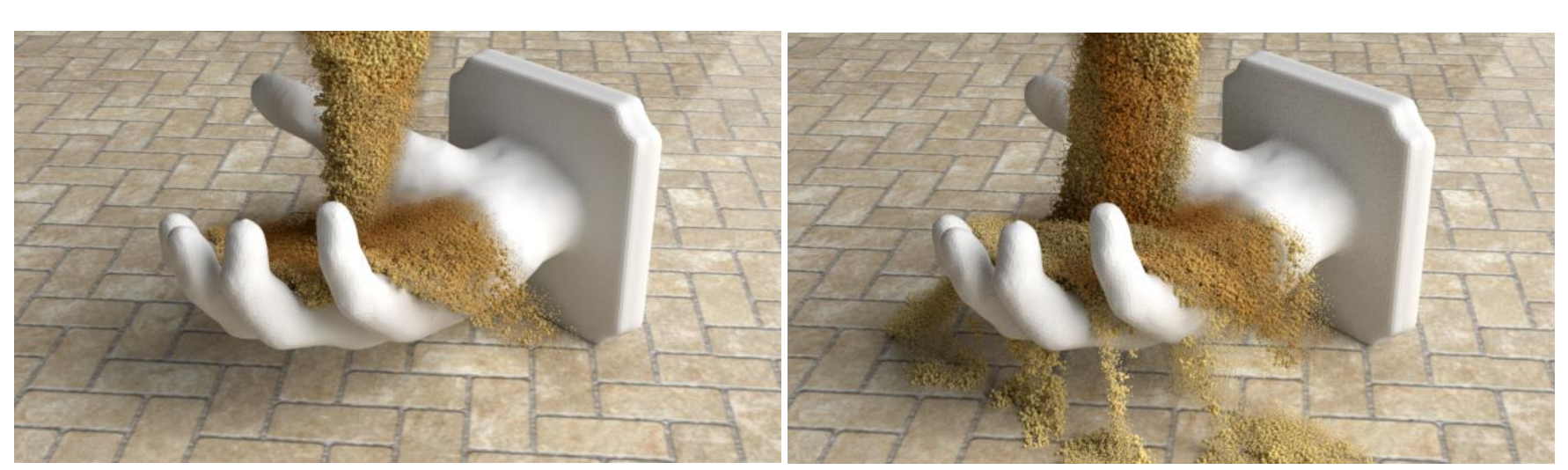

Figure 1: Powdered material interacting with a hand model. Powdered material has been thrown into the scene with a static hand model, granular particles, dust smog, dust particles coexist during the simulation.

\begin{abstract}
Powdered materials, such as sand and flour, are quite common in nature, whose properties always range from granular particles to smog materials under the air friction while throwing. This paper presents a hybrid method that tightly couples APIC solver with density field to accomplish the transformation of continuous powdered materials varying among granular particles, smog, powders and their natural mixtures. In our method, a part of the granular particles will be transformed to dust smog while interacting with air and represented by density field, then, as velocity decreases the density-based dust will deposit to powder particles. We construct a unified framework to imitate the mutual transformation process for the powdered materials of different scales, which greatly enhance
\end{abstract}

${ }^{*}$ Y. Gao and Y. Xu contributed equally and should be considered co-first authors.

Permission to make digital or hard copies of all or part of this work for personal or classroom use is granted without fee provided that copies are not made or distributed for profit or commercial advantage and that copies bear this notice and the full citation on the first page. Copyrights for components of this work owned by others than ACM must be honored. Abstracting with credit is permitted. To copy otherwise, or republish, to post on servers or to redistribute to lists, requires prior specific permission and/or a fee. Request permissions from permissions@acm.org.

VRST '19, November 12-15, 2019, Parramatta, NSW, Australia

(c) 2019 Association for Computing Machinery.

ACM ISBN 978-1-4503-7001-1/19/11 ..\$15.00

https://doi.org/10.1145/3359996.3364266 the details of particle-based materials modeling. We have conducted extensive experiments to verify the performance of our model, and get satisfactory results in terms of stability, efficiency and visual authenticity as expected.

\section{CCS CONCEPTS}

- Computing methodologies $\rightarrow$ Physical simulation.

\section{KEYWORDS}

Granular Material, Density Field based Smog, Two-Way Transformation, APIC

\section{ACM Reference Format:}

Yang Gao, Yinghao Xu, Shuai Li, Aimin Hao, and Hong Qin. 2019. A Hybrid Method for Powdered Materials Modeling. In 25th ACM Symposium on Virtual Reality Software and Technology (VRST '19), November 12-15, 2019, Parramatta, NSW, Australia. ACM, New York, NY, USA, 10 pages. https: //doi.org/10.1145/3359996.3364266

\section{INTRODUCTION AND MOTIVATIONS}

In recent years, more and more researchers begin to focus on the vivid details of natural phenomena which are usually ignored by commonly-used models. Powdering is an interesting and common phenomenon in real world, which contains a variety of different 
sizes of particles varying from coarse granules to fine dust particles. Of which, fine-grained dust particles may not follow other coarse particles during throwing, due to the small size and light weight, fine-grained dusts are more affected by air friction to float as smog, and then gradually settle on the floor. There are many state-of-the-art researches focus on granular material simulations, these particle-based materials usually refer to large aggregates of tiny solid grains with a diameter of $100 \mu \mathrm{m}$ or more [Andreotti et al. 2013], and most of the individual particles exhibit rigid properties. The most common granular material like sand can be simulated as fluid motion. When the particles are stationary, sandpile is formed according to its static friction coefficient, which is the difference between granular material and fluid. For most of the existing researches, granular material is usually simulated as single kind of particles with constant size, the classical methods include SPH [Yan et al. 2016] , PBD [Macklin et al. 2014], FLIP/PIC [Jiang et al. 2015; Zhu and Bridson 2005] and MPM [Daviet and BertailsDescoubes 2016; Tampubolon et al. 2017]. However, few studies concerns about reconstructing such a complicated process with multiple materials yet, one of the most important challenges is how to interactively simulate particles, smog-like dusts and their natural inter-transitions simultaneously.

Powdered materials are similar to particle-based materials but have more complex components. The grain size of powdered materials is usually between $30 \mu \mathrm{m}$ and $100 \mu \mathrm{m}$, which is not a constant but changes due to particle dispersion and aggregation, analogous to charge accumulation phenomenon. Typical materials are flours with diameter between $15 \mu \mathrm{m}$ and $40 \mu \mathrm{m}$, fine-grained sand and dust in sandstorms with diameter around $70 \mu m$, etc. Such kind of material, when not affected by air or wind, exhibit sand-like properties: accumulating and flowing on the ground as elasto-plastic or rigidplastic flow [Daviet and Bertails-Descoubes 2016]. However, when powdered material is thrown, particles of different sizes will exhibit different kinematic behaviors, heavy particles move fast while light dusts particles are affected by air friction to float as smog, this motion process is hard to be simulated with single kind of particles. From a simplified perspective, the diameter of particles occupying the bulk of material determines whether the property tends to be like granular material or fog material.

To reproduce such a dust-blowing phenomenon, we aim to model the vivid moving process of powder materials. We consider that the behavior of powdered material is analogous to that of particle material to some extent, which is effected mainly by contact and dry frictions, and as a highly dissipative system the motion procedure exhibits various physical states. And the behavior of tiny dust is analogous to smog when affected by the air friction, so we choose density field to represent the dust smog. Our hybrid model is built upon the flexible and effective intertwinement of the particles system and the grid density field, wherein the distributions and transitions of the granular particles are handled at geometry level while the dynamics of two models are tightly coupled at physics level. Generally speaking, the salient contributions of this paper can be summarized as follow.

- We propose a novel hybrid framework to model powdered materials and their natural mixtures of multi-scale particles and dust-like smog, which can enable physics-meaningful detail enhancement via mass-conserving smooth transition among large particles, smog, and tiny particles.

- We formulate an efficient APIC-Density field model to simulate the interaction and transition among different scales of granular particles and dust smog by introducing the rigorous particle-smog drag force definition.

\section{RELATED WORKS}

Mixing both particle approach and grid methods allows to keep the high-resolution details from particles while relying on a background mesh to reduce the number of DoF (degrees of freedom) of the system, as well as its computational cost. Foster and Metaxas first introduced PIC techniques to computer graphics with liquid simulation by solving the N-S equation on the grid, and used the labeled particles to track the fluid elements [Foster and Metaxas 1996]. Enright et al. proposed a Particle Level Set (PLS) method, adding particles on both sides of the fluid surface, along with the level set function, and updating it driven by the grid velocity field, to compensate the lack of detail when the lower-level set function identifies the fluid surface [Enright et al. 2002]. Zhu and Bridson popularized the widely-used linear combination of FLIP and PIC [Zhu and Bridson 2005], and Later Bridson et al. developed a number of extensions, including improved treatment of boundary conditions in irregular domains and coupling with rigid bodies [Batty et al. 2007], viscosity treatment [Batty and Bridson 2008], DiscontinuousGalerkin-based adaptivity [Edwards and Bridson 2014], multiphase flow [Boyd and Bridson 2012] and higher-order accuracy [Edwards and Bridson 2012]. Then, Cornelis et al. [Cornelis et al. 2014] coupled high-resolution FLIP with a low-resolution implicit Smoothed Particle Hydrodynamics (SPH) from Ihmsen et al. [Ihmsen et al. 2013]. Gerszewski and Bargteil used mass-full FLIP with a unilateral incompressibility constraint to resolve large-scale splashing liquids [Gerszewski and Bargteil 2013]. Stomakhin et al. chosen to let the particles carry more properties (such as mass and temperature) and used Material Point Method (MPM) method to couple the particles with the grid for snow simulation [Stomakhin et al. 2013]. Later Stomakhin et al. extended this method to simulate melting and freezing [Stomakhin et al. 2014]. Chentanez et al. coupled 3D Euler mesh, particle and height field method to simulate large-scale fluid scenes, simultaneously meeting the real-time interactivity of fluid detail retention and simulation [Chentanez et al. 2015]. After that, Ferstl et al. proposed a Narrow-Band FLIP simulation method, which greatly reduced the number of particles needed to maintain, and improved the FLIP simulation efficiency while maintaining almost the same visual effect [Ferstl et al. 2016].

Jiang et al. proposed Affine Particle in Cell modle (APIC) recently, which achieves stability conservation when doing transfer in Lagrangian/Eulerian hybrid methods to avoid dissipation and energy loss at the same time, it has been applied to the simulation of a variety of materials [Jiang et al. 2015]. Ram and Gast et al. introduced a new MPM-based model into the APIC method to simulate viscoelastic fluids, foams and sponges, and achieved good results [Ram et al. 2015]. Daviet and Bertails et al. also successfully used APIC to achieve continuous simulation of granular material, and the simulation effect is very realistic [Daviet and Bertails-Descoubes 2016]. Then, Frost et al. created a proprietary APIC fluid solver for various 
water simulations, and applied related technologies to animation production to get realistic visual effect [Frost et al. 2017]. Compared to popular FLIP/PIC models, APIC can produce more smooth and stable surfaces while simultaneously resolving more energetic fluid details than pure FLIP methods.

Many researchers have been successfully simulated granular materials. Bell et al. proposed a method for simulating granular materials based on Lagrangian particle representation [Bell et al. 2005]. Hybrid methods were first applied to granular materials in computer graphics by Zhu and Bridson [Zhu and Bridson 2005], they used a unified FLIP/PIC framework to handle fluid and sand behaviors. Narain et al. extended this approach to free-flowing materials subjecting to a maximum volume fraction constraint, and used a staggered LCP method to account for the coupling between pressure and frictional stress [Narain et al. 2010]. Stomakhin et al. designed an elastoplastic model for snow simulation [Stomakhin et al. 2013], which was later adjusted by Jiang et al. to simulate sand [Jiang et al. 2017]. Daviet et al. proposed a semi-implicit method for continuous simulation of granular materials based on APIC, which is better for large time steps [Daviet and Bertails-Descoubes 2016]. After that, Tampubolon et al. proposed a multi-category model for simulating the interaction of gravity-driven landslides and debris flows with porous sand materials and water, and realized the realistic modeling of sand behavior with different humidity [Tampubolon et al. 2017]. Gao et al. proposed a hybrid semi-implicit MPM method to simulate the deposition of particle flows, they use two MPM background grids to handle fluid and sediment two-way coupling, and achieved good performance [Gao et al. 2018]. To conclude, simulations of powdered/granular materials and their interactions have achieved great success, most of the previous works simulated the granular materials as a single component, ignoring their possibly complex compositions and motion behaviors.

In real world, powder materials may contain a variety of tiny, lightweight smog particles, which may also be generated by granular particles when interacting with air and other particles during motion. In 2007, Liu et al. proposed a physics-based approach to simulate sandstorms [Liu et al. 2007], using a relatively stable incompressible multi-fluid model to simulate the motion of air, sand and dust particles. Later in 2008, they simulated a two-fluid phenomenon consisting of gas flow (wind field) and dust particle flow in a unified framework based on a Reynolds average two-fluid model (RATFM) [Liu et al. 2008]. Wang et al. proposed a physics-based modeling and simulation method that can be used to synthesize various modes of sand [Wang and $\mathrm{Hu}$ 2012]. And Yang et al. proposed a new hybrid Lagrangian-Eulerian model to simulate the behavior of multi-scale droplets and mist spray mixtures [Yang et al. 2014], which simulated water spray with density field and built a unified transition system between water, droplet particles and water spray.

Strongly motivated by the idea of modeling particles-density field system [Yang et al. 2014], we aim to simultaneously simulate individual granular particles and smog-like density field, as well as their transformations to realize the visual-detail enhancement of powdered materials, and accommodate physical correctness and ensure the high-effective at same time.

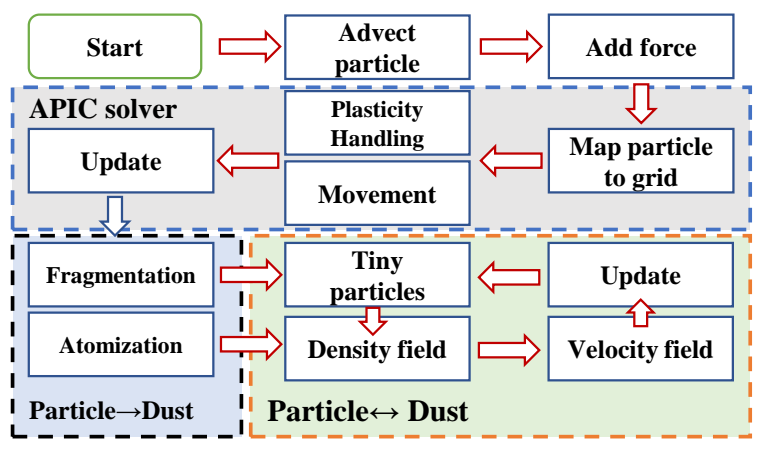

Figure 2: The framework of our model.

\section{METHOD OVERVIEW}

The framework of our model is shown in Fig. 2. Our hybrid model consists of two tightly-integrated parts: granular-materials-based particle solver and dust-smog-based grid solver. The particle-based simulation is solved by an APIC solver, which is briefly introduced in Section 3.2. Though both fluid and powdered materials are modeled by APIC, a major difference distinguishing particles from ordinary fluid is the contact and dry friction, details are explained in Section 4.1. We employ Eulerian based representation and solver to simulate dust smog generated by atomization of granular particles, detailed algorithm is described in Section 4.2.

\subsection{Pipeline}

Another important part of our model is the transition between particle and smog. As indicated with red arrows in Fig. 3, the transition between smog and tiny dust particles is bilateral. For sand particles, breaking into dust particles with smaller diameter occurs at positions where the movements and collisions are violent, or the contact with air is so drastic that the particles are affected more by the air and then split into smog. This process includes mass conservation, which is described in Section 5.1. As for dust smog, once the density reaches to a threshold or the movement becomes stagnant, dust suspended in the air gather together into small dust particles, the process is also referred to dust accumulation phenomenon, detailed in Section 5.2 .

\subsection{APIC Review}

Our hybrid model builds upon on APIC method, we employ this fluid solver for granular and tiny particles. Of which, kinematic steps are done on particles and dynamic steps are done on the grid, which is also shown in Fig. 3. Fluid dynamics is essentially based on Navier-Stokes (N-S) equations, which conserve both mass and momentum:

$$
\begin{gathered}
\frac{\partial \rho}{\partial t}+\rho \nabla \cdot \mathbf{u}=0 \\
\rho\left(\frac{\partial \mathbf{u}}{\partial t}+\mathbf{u} \cdot \nabla \mathbf{u}\right)=-\nabla p+\mu \nabla^{2} \mathbf{u}+\mathbf{f},
\end{gathered}
$$

where $\rho$ represents the density, $\mathbf{u}$ represents particle velocity, $p$ represents the pressure, and $\mathbf{f}$ represents the external force. 
For APIC, the fluid volume is represented and calculated by particles, and the N-S equations are solved with traditional Eulerian method. The velocity, rather than the velocity change, is interpolated over particles in APIC. However, there is a most crucial improvement: three vectors representing 3D affine velocity fields are stored on every particle, making APIC different from PIC method. Combined with this improvement, APIC method is able to avoid not only the dissipation that PIC suffers from, but also the noise and unstable behaviors that appear in FLIP. Thus, in APIC, the exact conservation of angular momentum across the transfers between particles and grid is available. This benefits a lot to our simulation of powdered material, whose tangential and rotational motion affect the simulation result so much.

Staggered mesh has been used in our fluid simulation, we transfer properties from particles to grids using

$$
\begin{gathered}
m_{a i}^{n}=\sum_{p} m_{p} \omega_{a i p}^{n}, \\
m_{a i}^{n} u_{a i}^{n}=\sum_{p} m_{p} \omega_{a i p}^{n}\left(\mathbf{e}_{a}^{T} \mathbf{u}_{p}^{n}+\left(\mathbf{c}_{p a}^{n}\right)^{T}\left(\mathbf{x}_{a i}-\mathbf{x}_{p}^{n}\right)\right),
\end{gathered}
$$

where subscript $a$ represents $x, y$ or $z$ directions, subscript $p$ and $i$ represents particle $p$ and grid node $i$, and $\mathbf{e}_{a}$ represents the unit vector in $a$ direction. And when interpolating back, we transfer from grids to particles by

$$
\mathbf{u}_{p}^{n+1}=\sum_{a, i} \omega_{a i p}^{n} \tilde{u}_{a i}^{n+1} \mathbf{e}_{a}
$$

and

$$
\mathbf{c}_{p a}^{n+1}=\sum_{i} \nabla \omega_{a i p}^{n} \tilde{u}_{a i}^{n+1}
$$

In equation 4 , the weights are $\omega_{\text {aip }}^{n}=N\left(\mathbf{x}_{a i}-\mathbf{x}_{p}^{n}\right), N(\mathbf{x})$ is the common trilinear interpolation kernel, and $\mathbf{x}_{a i}$ is the location of a MAC grid associated with direction $a . m_{a i}^{n}$ and $\mathbf{u}_{a i}^{n}$ are the mass and velocity component on the MAC grid, and $\mathbf{c}_{p a}^{n}$ is the affine vector per axis.

As the simulation using MAC grid, particles are endowed with mass $\mathbf{m}_{p}$, position $\mathbf{x}_{p}$, velocity $\mathbf{u}_{p}$ and three vectors $\mathbf{c}_{p a}$ representing affine states. Relatively, properties conserved on grid are mass $\mathbf{m}_{a i}$ (scalar), position $\mathbf{x}_{a i}$ and velocity $\mathbf{u}_{a i}$. And for solid or rigid bodies, a full affine representation of the local grid data $\mathbf{B}_{p}$ has been used instead of $\mathbf{c}_{p a}$.

\section{HYBRID MODEL FOR PARTICLES AND SMOG}

In this section, we will expound the two modeling parts of our model, Lagrangian-particle-based particles and Eulerian-grid-based smog. We modify the regular APIC fluid solver to handle solid particles movements [Jiang et al. 2015], with which we are able to simulate granular and dust particles with reasonable physical behaviors. As for smog simulation, we acquire density and velocity distributions transforming from sand particles as initialization when smog field has been generated, and the density should satisfy the transition condition meeting mass conservation. Then as well as smog density field within grid cells satisfy the accumulation condition, quantities accumulate and tiny dust particles appear and fall down.

\subsection{Yielding Condition for Granular Material}

To simulate behaviors of sand-like granular particles, we add a external operating part right after projection step on grid through the following steps:

First, we evaluate the strain rate tensor $\mathbf{D}$ in each grid cell with standard central differences by

$$
\mathbf{D}=\left(\nabla \mathbf{u}+\nabla \mathbf{u}^{T}\right) / 2,
$$

in which $\nabla \mathbf{u}$ is the gradient of velocity field, and $\nabla \mathbf{u}^{T}$ represents its transposition.

Then we calculate the frictional stress, wherein the particles are flowing:

$$
\sigma_{f}=-\sin \Phi p \frac{\mathbf{D}}{\sqrt{1 / 3}|\mathbf{D}|_{F}} .
$$

Here $\Phi$ is the friction angle, $p$ is the pressure computed in projection step, and $|\mathbf{D}|_{F}$ is the Frobenius norm of $\mathbf{D}$. As for static granular particles, the frictional stress is

$$
\sigma_{\text {rigid }}=-\frac{\rho \mathbf{D} \Delta x^{2}}{\Delta t}
$$

which means the minimum stress required for rigidified particles between two grid cells. Thus, we can determine yielding condition for each cell by comparing the two stress:

$$
\sigma_{f}<\sigma_{\text {rigid }}+c,
$$

where $c>0$ is the cohesion coefficient, and it is set to adjust cohesion and avoid slippage. When the yielding condition is satisfied, we then mark the grid cell as rigid and store $\sigma_{\text {rigid }}$ at that location. Otherwise, we store the sliding frictional stress $\sigma_{f}$.

Then the two regions are treated respectively: for rigid cells, we find all connected groups of rigid cells that are just marked, and project the velocity field in each separate group to the space of rigid body motions:

$$
\mathbf{u}(\mathbf{x})=\overline{\mathbf{u}}+(\mathbf{x}-\overline{\mathbf{x}}) \times \bar{\omega},
$$

where $\overline{\mathbf{x}}$ represents the centroid of the connected group, $\overline{\mathbf{v}}$ represents the average velocity, and $\bar{\omega}$ represents the average angular velocity. As for all the non-rigid cells, we update velocity by

$$
\mathbf{u}=\mathbf{u}^{\prime}+\frac{\Delta t}{\rho} \nabla \cdot \sigma_{f}
$$

where $\sigma_{f}$ is the stored frictional stress. Moreover, we also change general boundary condition to frictional boundary condition. With the velocity adjustment by using yielding condition, we turn a common APIC fluid solver into a continuum solver for granular particles.

\subsection{Dust Smog Modeling}

As for the simulation of density-based dust smog, we employ Eulerian based representation. We compute the density field for transferring particles, whose velocity satisfies the criteria for smog generation (Section 5.1). When smog generated, we delete the same amount of particles and emit smog field at the same position simultaneously. Conservation condition is ensured through the same initial value of density and velocity field. The velocity field of smog density field $\mathbf{u}_{d}$ is decided by 


$$
\rho\left(\frac{\partial \mathbf{u}_{d}}{\partial t}+\mathbf{u}_{d} \cdot \nabla \mathbf{u}_{d}\right)=-\nabla p+\mathbf{f},
$$

where $\mathbf{f}$ is additional force. In our model, we replace this additional force with air resistance as a user defined force. Then, we govern the density field by

$$
\frac{\partial \rho}{\partial t}+\mathbf{u}_{d} \cdot \nabla \rho=\mu \nabla^{2} \rho-d \rho+S
$$

where $\mu$ represents the diffusion coefficient, $d$ represents the dissipation coefficient, and $S$ is the surrounding area in smog source.

It should be noted that, when we use Eulerian method to simulate smog-like material, since the material component usually has quite little mass and volume, which is greatly affected by the air buoyancy. The dust smog will settle into small tiny particles under gravity force in a long enough time period. Thus, we take gravity as an external forces and apply it to dust smog during simulation.

\section{TRANSITIONS BETWEEN PARTICLE AND DENSITY FIELD}

In this section, we expound our two-way transitions between particle state and smog state of powdered material, focusing on the transition condition and material conservation.

\subsection{Smog Generation from Particles}

For powdered materials, in the case of high-speed movement, due to air resistance and some other complex forces, the large granular particles will break into smaller particles as tiny dusts. Moreover, if a particle's velocity changes rapidly, the granular particles will also be converted to 'smog' state directly. We simply use the particle velocity and relative velocity between one particle and its surrounding particles:

$$
\mathbf{u}_{p}^{r e l}=\left|\mathbf{u}_{p}-\frac{\sum_{i \in S} m_{i} \omega_{i} \mathbf{u}_{i}}{\sum_{i \in S} m_{i} \omega_{i}}\right|,
$$

where $S$ is the surrounding area in neighbor cell of particle $p$, and $\omega_{i}$ is a smooth weighting kernel.

The conversion of particle to density field occurs only on the interface between particles and air or nearby positions. In other world, we need to judge if at least one air cell exists near by a solid particle. However, if one granular particle inside satisfies all the generation condition, it will not be transferred into density field of smog, because the air pressure around this particle is nearly constant, which means there are not enough driving forces to levitate smog, instead, it will be broken into smaller particles with smaller diameter as tiny dust particles directly, the velocity of tiny dust is same to its father granular particle and its mass is defined as:

$$
m_{d i}=a \cdot m_{i}, a \in[0,0.5] .
$$

Here $m_{d i}$ is the mass of dust particle, $a$ is a parameter relate to the magnitude of velocity which should be divisible by 1 , the generated number of dust particles is $1 / a$ so the dust generation process satisfies mass conservation, since $a \in[0,0.5]$, it means there are two dust particles will be generated at least. That is, we judge for a deleted-particle whose neighbor cells have at least one air cell for density field generation, otherwise it will split to tiny dust particles.
Considering another situation when the overall movement of the powdered material is intense, the relative speed in this area may not be large, but the overall movement speed may also generate sufficient driving force to promote the transition. We set an absolute speed threshold $\mathbf{u}_{\text {thres }}^{\max }$ as another condition for speed conversion. When the transition occurs, in order to satisfy the mass conservation, we convert the particles into smog field of the same quality and corresponding density, and select adjacent cells near the position of the particles according to the diffusion coefficient, distribute the values proportionally to the density field of these cells with a trilinear interpolation function. Our transition process is developed from the handling for spray-droplet transitions of Yang et al. [Yang et al. 2014].

In short, the transition from particle state to dust smog has to satisfy two conditions: the first one is that the particle should be located at the surface of the solid set or closed to the surface cell (e.g., the position is in contact with air). The second is that the difference between the velocity of the particle and its average velocity reaches the relative velocity threshold $\mathbf{u}_{p}^{r e l}$ or its absolute velocity reaches the absolute velocity threshold $\mathbf{u}_{\text {thres }}^{\max }$. When only the second condition is satisfied, granular particle will fragment to dust particles instead of smog. It should be noted that, both large granular particles and tiny dust particles can generate density field of dust smog, the only difference between them is that large granular particles inside may transform to dust particles, but vice versa will not happen.

\subsection{Transformation between Smog and Dust Particles}

In real world, flour will eventually fall to the ground and sandstorms without wind will also turn into dust piles, so we design a two-way transformation framework to imitate this physics-based process of dust accumulation.

Static smog will generate dust particles, wherein many tiny dust particles are clustered in a tiny area, the attraction effect for each other is similar to electrostatic attraction. These particles will aggregate into one or more general dust particles. This phenomenon will occur when the density of one grid cell reaches a threshold:

$$
\rho_{\text {thres }}=\alpha N \bar{\rho} \text {. }
$$

where $N$ is the number of cells affected when a dust particle is transferred, $\bar{\rho}$ is the density added to the grid cell when this particle is transferred to smog, and $\alpha$ is a conversion coefficient used to control the conversion threshold, which represents the density value obtained by converting $\alpha$ particles into this grid cell. $\alpha>\frac{1}{N}$ should be satisfied to avoid the repeated and meaningless transition within a single time step that particle transfers into dust smog and dust smog immediately reverses back to particle. Here we set $\alpha=1$, which facilitates mass conservation and generates particles. However, if the density reaches $\rho_{\text {thres }}$ and the dust smog is still moving at a high speed, the transition will not happen in this case, such as sandstorm. Therefore, it is also necessary to set a minimum speed threshold $\mathbf{u}_{\text {thres }}^{\text {min }}$ to handle this situation, which means the upper bound of the speed at which the transition occurs.

In another case, as the movements weakens, the velocities of all dust smog tends to be $\mathbf{0}$, which means dust smog tends to be static, 


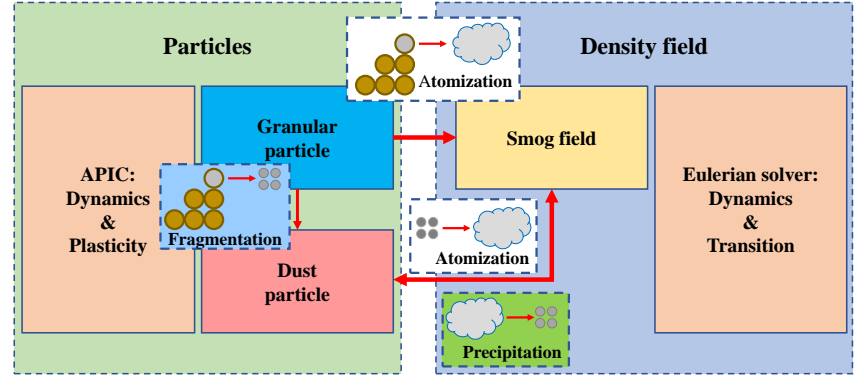

Figure 3: The conversion relationship among granular particles, dust smog and dust particles.

here we use another condition. Since the final state of powdered material is definitely similar to granular material, that is, immobile particles on the ground instead of suspended smog in the air. So we transfer all possible smog fields to dust particles when their velocities are below precipitation threshold of $\mathbf{u}_{\text {thres }}^{\text {stop }}$, the threshold we set for low-velocity dust particle generation is also the minimum velocity that smog should carry. For those whose velocities are less than $\mathbf{u}_{\text {thres }}^{\text {stop }}$, we consider them as dust particles-based materials that will precipitated. Through this unified measurement, we can realize dust accumulation with physical meaning inspired by reality.

In addition, the diffused density field of some grid cells may not enough to accumulate into dust particles, it can be regarded as discrete tiny dust particles that is too small to be observed by naked eyes. As a result, we consider very few of dust smog as discrete parts and ignore them. To explain this in reality, powdered materials can hardly remain all quantities after movements to some extent, so it is reasonable to regard a small part of smog as discrete part that is dissipated. This lower density limit used here is referred to the dissipative density $r h o_{d i s}$ to remain global conservation.

To conclude, there are two conditions for the transition from density field to the particle state: first, the density of the cell is greater than $r h o_{\text {thres }}$ and the cell velocity is less than $\mathbf{u}_{\text {thres }}^{\text {min }}$, that is, the dense dust smog has low speed. Second, the cell velocity is less than $\mathbf{u}_{\text {thres }}^{\text {stop }}$, and the cell density is greater than the dissipative density $r h o_{d i s}$, which means low-speed dust smog that cannot be ignored.

It also should be noted that, in our method the dust particles transferred directly from granular particles and generated from smog are solved by the APIC solver as well, but it is not the same as the original granular particles, the size of these particles is smaller than that of the original ones. They can generate smog field, and then be transferred back following the rules mentioned before. In order to distinguish the two different scales of particles, the converted tiny particles are marked as dust particles, whose mass is determined by the conversion threshold and the conversion density. During rendering, their particle sizes are set to be smaller than the original granular particles, visually appearing as a fine dusts pile. The complete conversion relationship is shown in Fig. 3, the transition from granular particles to dust (smog and tiny particles) is unidirectional and non-reversing, and dust smog and dust particles have two-way flexible transition rules, which is more like the real world scenarios.

\section{IMPLEMENTATION DETAILS}

In this section, we itemize all the steps of our method in a complete time step, and supplement the details of our implementation.

\subsection{Time Integration}

The main steps of our model within each time step is shown in Algorithm 1.

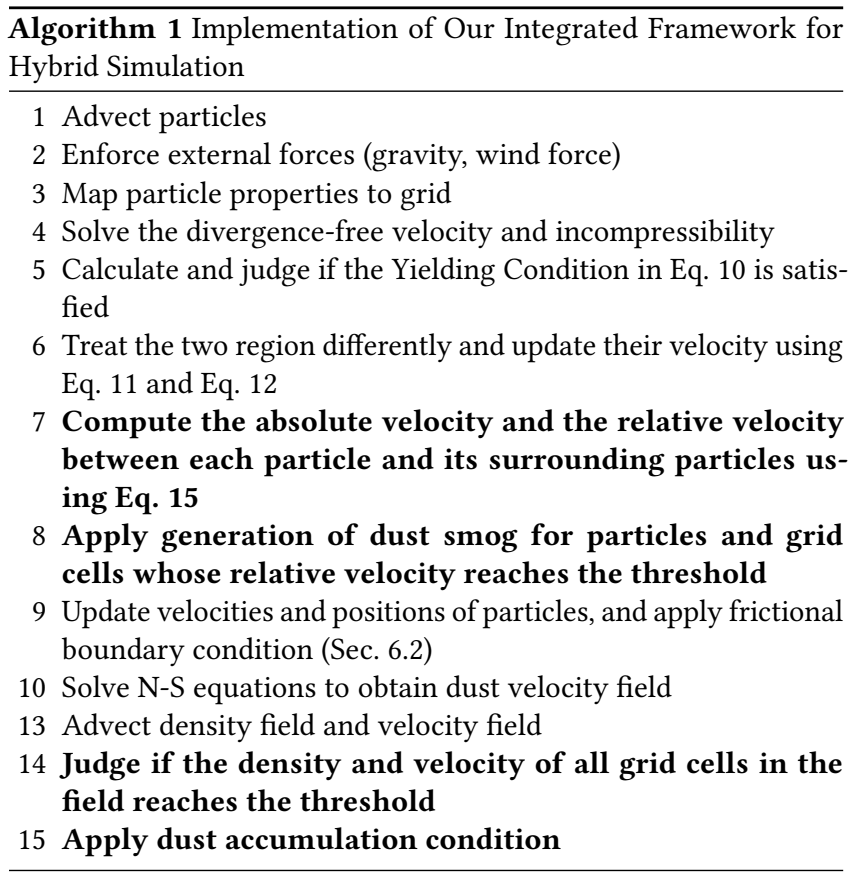

\subsection{Frictional Boundary Condition}

We adopt frictional boundary condition to cover the friction between powdered material and other objects with solid wall boundaries:

$$
\mathbf{u}_{T}=\max \left(0,1-\frac{\mu|\mathbf{u} \cdot n|}{\left|\mathbf{u}_{T}\right|}\right) \mathbf{u}_{T},
$$

where $\mu$ is the Coulomb friction coefficient between powdered material and boundary wall, $|\mathbf{u} \cdot n|$ is the normal velocity and $\mathbf{u}_{T}$ is the tangential velocity.

\section{EXPERIMENTAL RESULTS AND DISCUSSIONS}

Our hybrid particle-grid model has been implemented with $\mathrm{C}++$ and CUDA, while all experiments are run on a PC of $3.40 \mathrm{GHz}$ Intel Core i7 CPU and Geforce GTX1070 GPU. All the results are rendered by Blender (https://www.blender.org). And we design several scenarios to verify the effect and reality of the simulation model. Table 1 shows the performances and key parameters setting of our experiments, detailed parameters setting of Fig. 6 can be found 


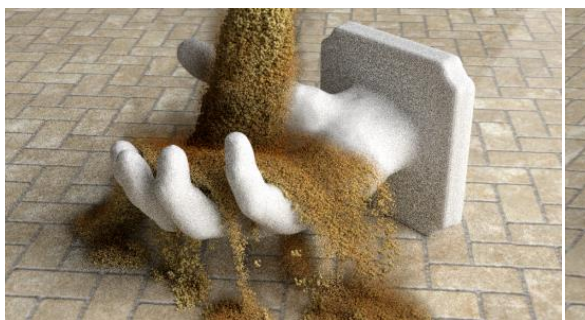

(a)

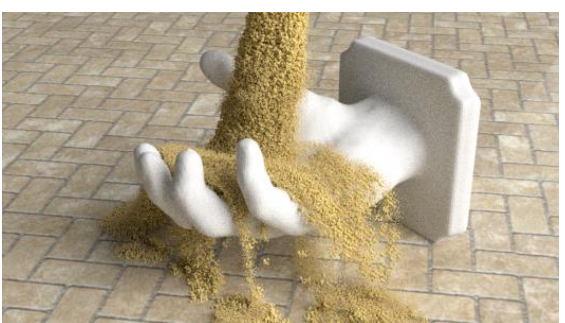

(b)

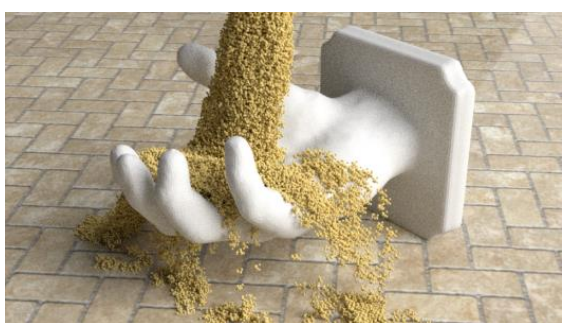

(c)

Figure 4: Comparisons of our hybrid model. (a) Powdered material simulation with granular particles, dust smog and dust particles; (b) Powdered material simulation with different scales of particles; (c) Granular materials simulation with single scale of particle.

Table 1: Parameters setting and time performances from the experimental results.

\begin{tabular}{|l|c|c|c|c|c|}
\hline Scenes & Resolutions & Particles & Time/Frame & $\mathbf{u}_{\text {thres }}^{\text {max }}$ & $\mathbf{u}_{p}^{\text {rel }}$ \\
\hline \hline Throwing (Fig. 6) & $64 \times 64 \times 64$ & $240 \mathrm{k}$ & $87.63 \mathrm{~ms}$ & - & - \\
Sandstorm (Fig. 9) & $64 \times 64 \times 64$ & $400 \mathrm{k}$ & $67.36 \mathrm{~ms}$ & 3.5 & 0.5 \\
Interaction 1 (Fig. 5) & $128 \times 64 \times 64$ & $450 \mathrm{k}$ & $163.17 \mathrm{~ms}$ & 1.0 & 0.3 \\
Interaction 2 (Fig. 7) & $128 \times 128 \times 64$ & $80 \mathrm{k}$ & $83.46 \mathrm{~ms}$ & 2.0 & 0.2 \\
Interaction 3 (Fig. 1) & $128 \times 128 \times 64$ & $150 \mathrm{k}$ & $131.88 \mathrm{~ms}$ & 2.0 & 0.2 \\
Explosion (Fig. 8) & $128 \times 128 \times 64$ & $960 \mathrm{k}$ & $187.09 \mathrm{~ms}$ & 4.3 & 0.8 \\
\hline
\end{tabular}

Table 2: Comparison of parameters Setting for Fig. 6 .

\begin{tabular}{|l|c|c|c|c|}
\hline Scene & $\mathbf{u}_{\text {thres }}^{\text {min }}$ & $\mathbf{u}_{\text {thres }}^{\max }$ & $\mathbf{u}_{\text {thres }}^{\text {stop }}$ & $\mathbf{u}_{p}^{\text {rel }}$ \\
\hline \hline Fig. 6 (b) & 10.0 & 100 & - & - \\
Fig. 6 (c) & 10.0 & 4.3 & 0.01 & 0.8 \\
Fig. 6 (d) & 10.0 & 1.0 & 0.01 & 0.2 \\
\hline
\end{tabular}

in Table 2. For all experiments in this table, $\mathbf{u}_{\text {thres }}^{\text {min }}=10.0$ and $\mathbf{u}_{\text {thres }}^{\text {stop }}=0.01$.

\subsection{Comparisons and Discussions}

Fig. 1 shows a scenario of interaction between powdered material and solid model. At the same time, we illustrate the other two comparisons (Fig. 4), one use particles model with different scales(Fig. 4 (b)) and the other only use traditional one scale of particle which has no transitions of both smog and dust (Fig. 4 (c)). It can be obviously seen from Fig. 4 (a), with our complete transition process, we can imitate more realistic details.

Fig.5 illustrates the results of our hybrid powdered materials model solved by different solvers. As powdered materials drop down under gravity force, a object moves forward and back quite fast to hit the particles. Fig.5 (1) shows the APIC solver we used for all our experiments [Jiang et al. 2015]. Fig.5 (b) displays the FLIP solver which makes particles and smog violently scattered. Fig.5 (c) shows a result solved by PIC solver that is too smooth to make the particle move very gently. It can be seen from these three scenarios that benefiting from APIC, the angular momentum is conserved during simulation, leading to better rotating and floating effects of powdered materials.

\subsection{Experiment Results}

This scene shows a throwing progress with different parameters (Fig. 6). A pile of powdered particles is thrown from center with an initial velocity and gravity, different transfer thresholds parameters, we can get different phenomena. Detailed parameter setting has been illustrated in Table 2 . There shows three behaviors of different degrees for smog generation, with extremely high threshold $\mathbf{u}_{\text {thres }}^{\max }=100$, particles will not transfer to dust, and the transfer degree can be flexibly controlled depends on different applications.

As shown in Fig. 7, powdered materials are dropped in the air, which interact with a static solid model in the air, and then fall to the ground. Smog field and tiny particles generated from large granular particles during the interactions. Since there is no external force driving, smog soon transfers to tiny particles, and all particles drop down under the gravity.

The powdered material in the middle of the scene is exploding from the center of the pile of particles, like a dust explosion in the scene. Particles fly in all directions, and the smog has been quickly generated.

In a $64 \times 64 \times 64$ sandbox, the powdered material has laid on the ground. Driven by an artificial tornado, sand particles are rolled into the air under the influence of a spiral wind field, like a sandstorm. As the movement becomes more intense, a lot of smog is generated and moves along with the sand. When the simulation progresses to a certain time point, the wind disappears and the powdered material hits the wall, and then slides back to the ground, dust smog gradually deposits as tiny dust particles and drop down to the ground. 

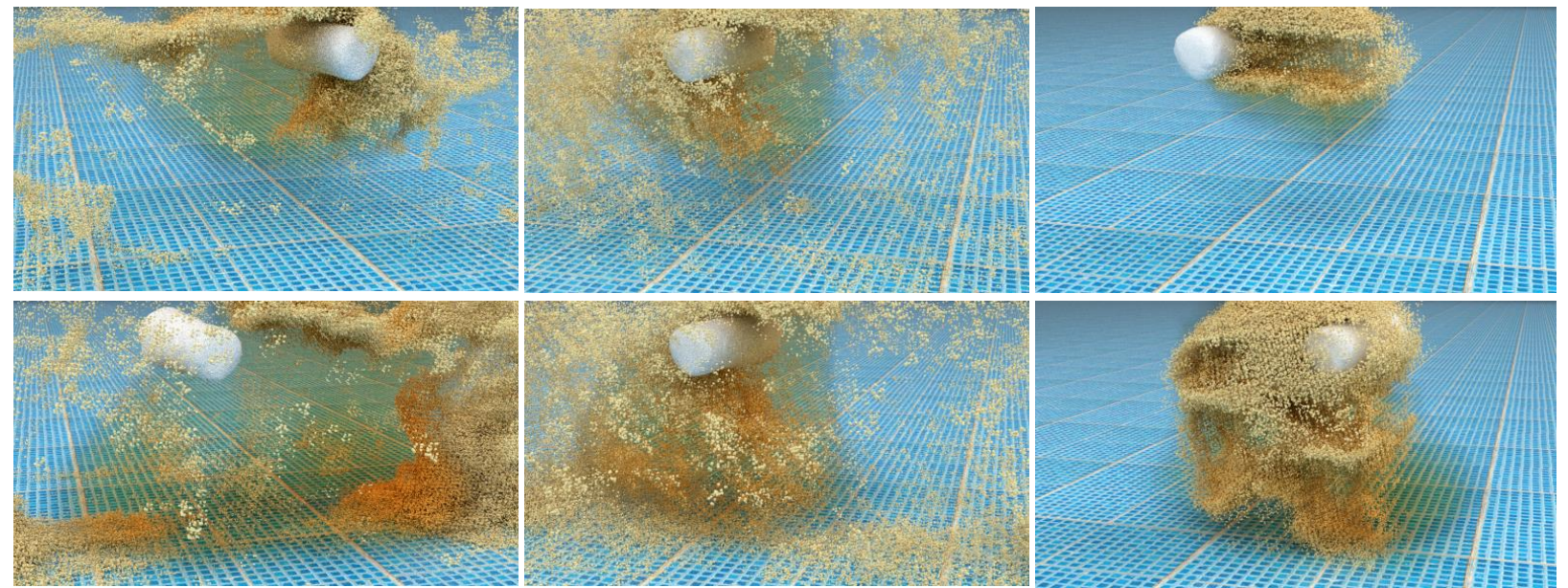

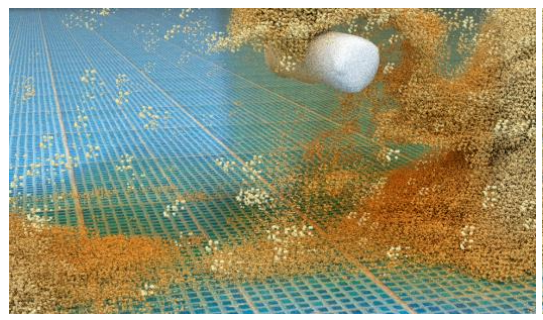

(a)

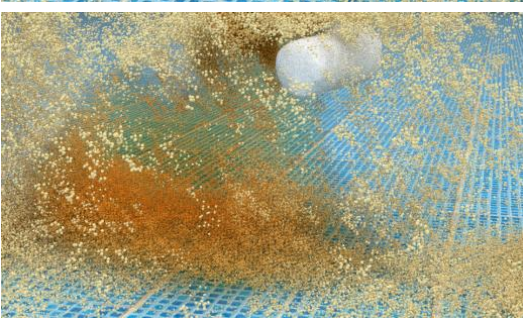

(b)

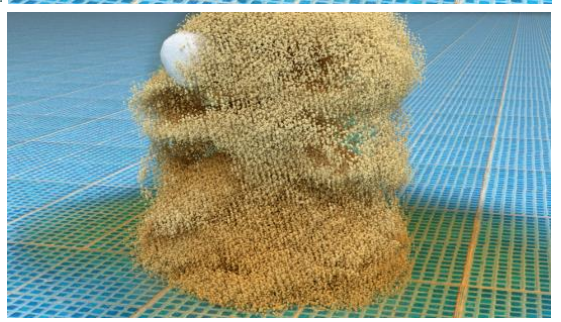

(c)

Figure 5: Comparisons of different solvers with our hybrid model at same frames (frames 150,250 and 350 ). A moving object hits the following powdered materials with different dynamic solvers: (a) Our APIC solver. (b) FLIP solver. (c) PIC solver.

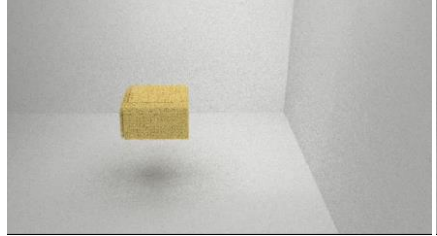

(a)

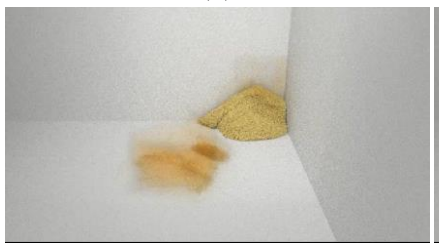

(c)

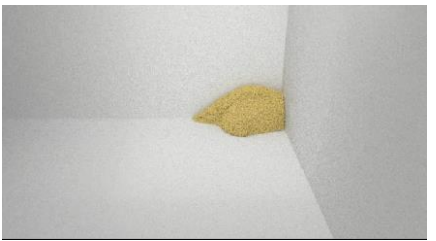

(b)

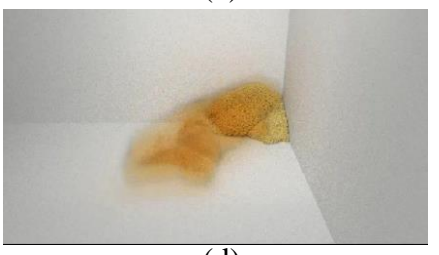

(d)
Figure 6: Throwing sand to a corner with different thresholds.(a) Initial state of this scene. (b) No transition from particles to smog. (c) A part of sand particles transforming to smog density.(d) A lot of smog generated from sand particles.

\section{CONCLUSIONS AND FUTURE WORK}

In this paper, we have presented a novel hybrid particle-grid model to simulate powdered material based on APIC method. Our model produces vivid visual effects for powdered materials in a critical state, which can not be realized by previous granular material simulation methods. By combining and coupling APIC and Eulerian methods, different states of powdered materials can be simulated simultaneously and flexibly transit to each other in a physics-based way. Our model is supportive to a variety of powder behaviors, including flowing on the ground, floating in the air and exploding with high velocity, and so on.

Our work may be the first attempt to model and simulate powdered material in a critical state with different materials. There are still some limitations cannot be ignored. Among the transformation from particles to smoke and smoke to particles, our simplified model considers only the inheritance of velocities. However, since the mass differences of different materials are quite huge and the generation of smoke density field is not strict conservation, our simulation is hard to ensure the global conservation of momentum.

\section{ACKNOWLEDGMENTS}

This research is supported in part by National Key R \& D Program of China (No. 2017YFF0106407), National Natural Science Foundation of China (NO. 61672077 and 61532002), Applied Basic Research 

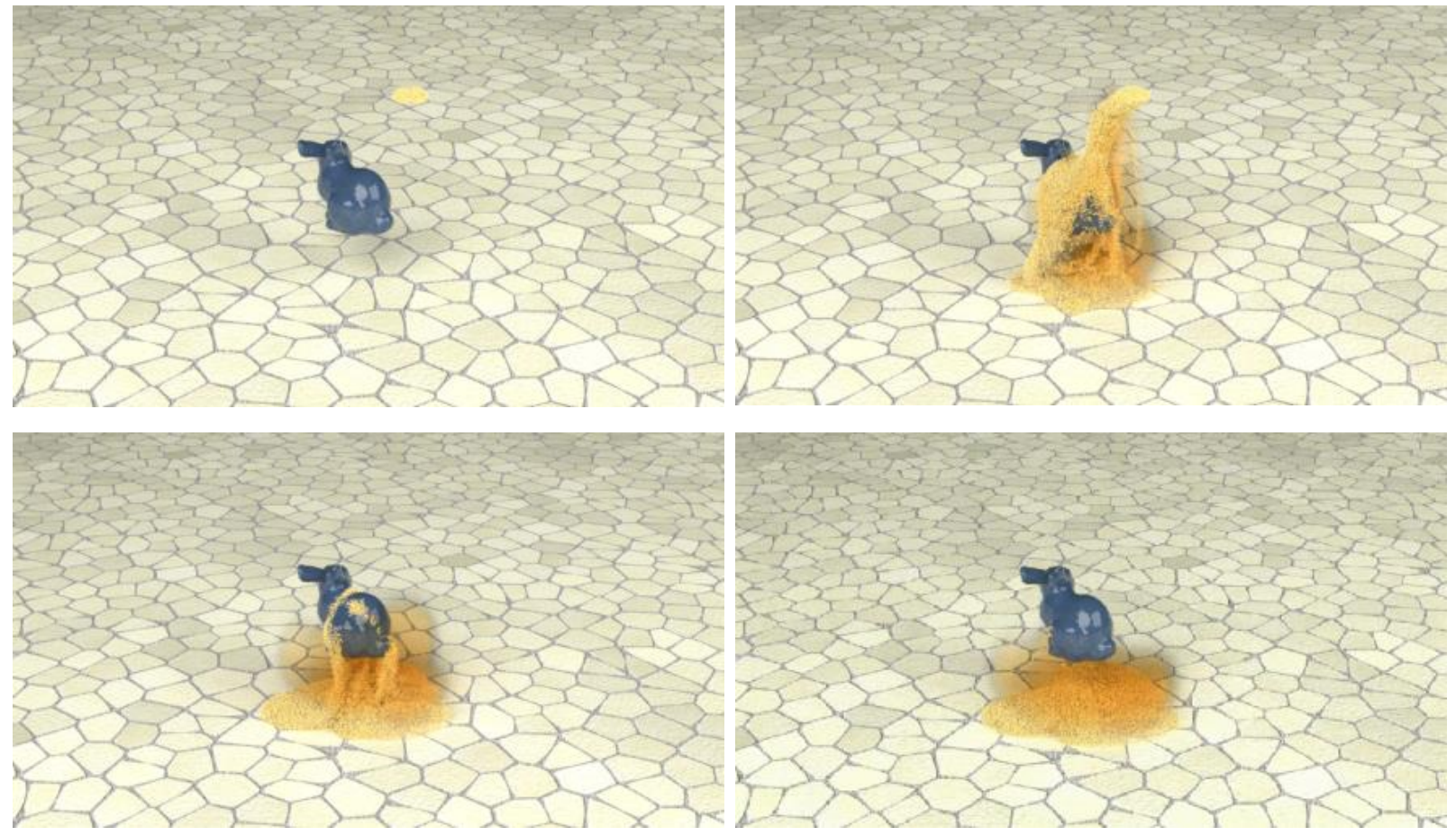

Figure 7: Pouring sand onto a solid model hanging in the air, sand particles interact with solid to generate dust smog and dust particles.
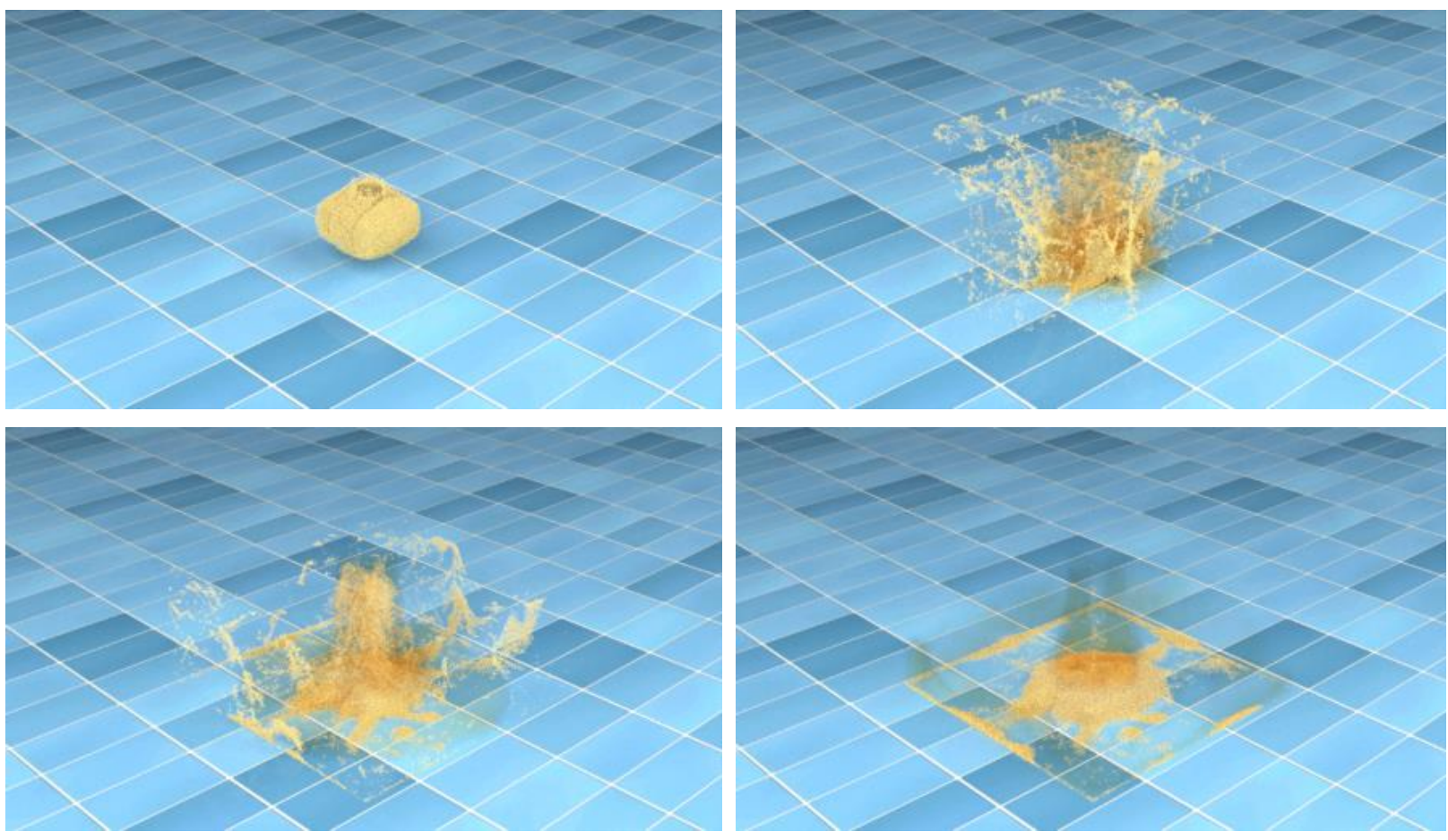

Figure 8: A cloud of grained sand-like materials explode in a sandbox. 

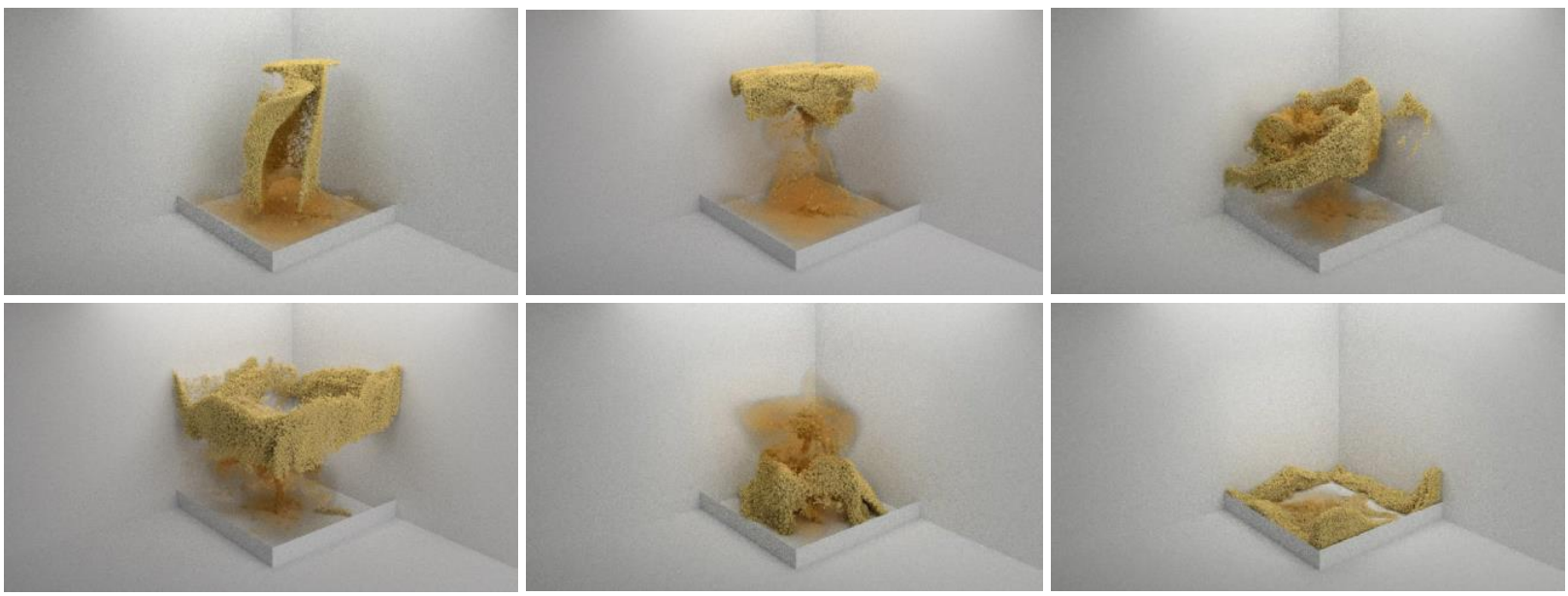

Figure 9: Sandstorm in a sandbox. Powdered material has been rolled into the air due to the spiral wind, dust smog and dust particles occur as the particles rotate, when wind force comes to 0 , the powdered material hits the wall boundary, and smog gradually deposits as dust particles and falls down.

Program of Qingdao (NO. 161013xx), National Science Foundation of USA (NO. IIS-0949467, IIS-1047715, IIS-1715985, and IIS-1049448), capital health research and development of special 2016-1-4011, and Beijing Natural Science Foundation-Haidian Primitive Innovation Joint Fund (L182016). We would like to thank the anonymous reviewers for paying time to raise constructive critiques to us.

\section{REFERENCES}

Bruno Andreotti, Yoël Forterre, and Olivier Pouliquen. 2013. Granular media: between fluid and solid. Cambridge University Press.

Christopher Batty, Florence Bertails, and Robert Bridson. 2007. A Fast Variational Framework for Accurate Solid-Fluid Coupling. ACM Transactions on Graphics (TOG) 26, 3 (2007).

Christopher Batty and Robert Bridson. 2008. Accurate viscous free surfaces for buckling, coiling, and rotating liquids. (2008), 219-228.

Nathan Bell, Yizhou Yu, and Peter J Mucha. 2005. Particle-based simulation of granular materials. In Proceedings of the 2005 ACM SIGGRAPH/Eurographics symposium on Computer animation. ACM, 77-86.

Landon Boyd and Robert Bridson. 2012. MultiFLIP for energetic two-phase fluid simulation. ACM Transactions on Graphics (TOG) 31, 2 (2012), 16.

Nuttapong Chentanez, Matthias Müller, and Tae-Yong Kim. 2015. Coupling 3D eulerian, heightfield and particle methods for interactive simulation of large scale liquid phenomena. IEEE transactions on visualization and computer graphics 21, 10 (2015), $1116-1128$.

Jens Cornelis, Markus Ihmsen, Andreas Peer, and Matthias Teschner. 2014. IISPH-FLIP for incompressible fluids. 33, 2 (2014), 255-262.

Gilles Daviet and Florence Bertails-Descoubes. 2016. A semi-implicit material point method for the continuum simulation of granular materials. ACM Transactions on Graphics (TOG) 35, 4 (2016), 102

Essex Edwards and Robert Bridson. 2012. A high-order accurate particle-in-cell method. Internat. 7. Numer. Methods Engrg. 90, 9 (2012), 1073-1088.

Essex Edwards and Robert Bridson. 2014. Detailed water with coarse grids: combining surface meshes and adaptive discontinuous Galerkin. ACM Transactions on Graphics (TOG) 33, 4 (2014), 136.

Douglas Enright, Ronald Fedkiw, Joel Ferziger, and Ian Mitchell. 2002. A Hybrid Particle Level Set Method for Improved Interface Capturing. F. Comput. Phys. 183, 1 (2002), 83-116.

Florian Ferstl, Ryoichi Ando, Chris Wojtan, Rüdiger Westermann, and Nils Thuerey 2016. Narrow band flip for liquid simulations. 35, 2 (2016), 225-232.

Nick Foster and Dimitri Metaxas. 1996. Realistic animation of liquids. Graphical models and image processing 58, 5 (1996), 471-483.

Ben Frost, Alexey Stomakhin, and Hiroaki Narita. 2017. Moana: performing water. (2017), 30.

Ming Gao, Andre Pradhana, Xuchen Han, Qi Guo, Grant Kot, Eftychios Sifakis, and Chenfanfu Jiang. 2018. Animating fluid sediment mixture in particle-laden flows.
ACM Transactions on Graphics (TOG) 37, 4 (2018), 149.

Dan Gerszewski and Adam W Bargteil. 2013. Physics-based animation of large-scale splashing liquids. ACM Trans. Graph. 32, 6 (2013), 185-1.

Markus Ihmsen, Jens Cornelis, Barbara Solenthaler, Christopher Horvath, and Matthias Teschner. 2013. Implicit incompressible SPH. IEEE transactions on visualization and computer graphics 20, 3 (2013), 426-435.

Chenfanfu Jiang, Craig Schroeder, Andrew Selle, Joseph Teran, and Alexey Stomakhin. 2015. The affine particle-in-cell method. ACM Transactions on Graphics (TOG) 34, 4 (2015), 51.

Chenfanfu Jiang, Craig Schroeder, and Joseph Teran. 2017. An angular momentum conserving affine-particle-in-cell method. F. Comput. Phys. 338 (2017), 137-164.

Shiguang Liu, Zhangye Wang, Zheng Gong, Lei Huang, and Qunsheng Peng. 2007. Physically based animation of sandstorm. Computer Animation and Virtual Worlds 18, 4-5 (2007), 259-269.

Shiguang Liu, Zhangye Wang, Gong Zheng, and Qunsheng Peng. 2008. Simulation of atmospheric binary mixtures based on two-fluid model. Graphical Models 70, 6 (2008), 117-124.

Miles Macklin, Matthias Müller, Nuttapong Chentanez, and Tae-Yong Kim. 2014. Unified Particle Physics for Real-time Applications. ACM Transactions on Graphics (TOG) 33, 4 (2014), 153:1-153:12.

Rahul Narain, Abhinav Golas, and Ming C Lin. 2010. Free-flowing granular materials with two-way solid coupling. 29, 6 (2010), 173.

Daniel Ram, Theodore Gast, Chenfanfu Jiang, Craig Schroeder, Alexey Stomakhin, Joseph Teran, and Pirouz Kavehpour. 2015. A material point method for viscoelastic fluids, foams and sponges. (2015), 157-163.

Alexey Stomakhin, Craig Schroeder, Lawrence Chai, Joseph Teran, and Andrew Selle. 2013. A material point method for snow simulation. ACM Transactions on Graphics (TOG) 32, 4 (2013), 102.

Alexey Stomakhin, Craig Schroeder, Chenfanfu Jiang, Lawrence Chai, Joseph Teran, and Andrew Selle. 2014. Augmented MPM for phase-change and varied materials. ACM Transactions on Graphics (TOG) 33, 4 (2014), 138.

Andre Pradhana Tampubolon, Theodore Gast, Gergely Klár, Chuyuan Fu, Joseph Teran, Chenfanfu Jiang, and Ken Museth. 2017. Multi-species simulation of porous sand and water mixtures. ACM Transactions on Graphics (TOG) 36, 4 (2017), 105.

Ning Wang and Bao-Gang Hu. 2012. Real-time simulation of aeolian sand movement and sand ripple evolution: a method based on the physics of blown sand. Fournal of Computer Science and Technology 27, 1 (2012), 135-146.

Xiao Yan, Yun-Tao Jiang, Chen-Feng Li, Ralph R Martin, and Shi-Min Hu. 2016. Multiphase SPH simulation for interactive fluids and solids. ACM Transactions on Graphics (TOG) 35, 4 (2016), 79.

Lipeng Yang, Shuai Li, Aimin Hao, and Hong Qin. 2014. Hybrid Particle-grid Modeling for Multi-scale Droplet/Spray Simulation. 33, 7 (2014), 199-208.

Yongning Zhu and Robert Bridson. 2005. Animating sand as a fluid. 24, 3 (2005), 965-972. 